\title{
Effets des termes non-linéaires dans les écoulements non-stationnaires à deux couches *
}

\author{
par M. Arhan et A. Cavanié \\ Centre Océanologique de Bretagne \\ C.N.E.X.O., Brest
}

\section{Introduction}

Les mesures de courant et de température en différents endroits, en pleine mer de même que sur les plateaux continentaux, ont mis en évidence des ondes internes se propageant au sein des eaux stratifiées de l'océan. Dans certaines régions, ces ondes ont des amplitudes telles qu'il paraît a priori difficile de négliger les termes non-linéaires dans les équations et les conditions aux limites qui gouvernent ces écoulements.

Les difficultés rencontrées pour résoudre le problème, dans le cas le plus général, conduisent à considérer un certain nombre de modèles particuliers, valables individuellement pour certaines échelles de temps et d'espace. Nous ne traiterons ici que d'écoulements plans, bornés par un fond et un plafond horizontaux imperméables, ce qui élimine les ondes de surface et impose que le débit volumique total des deux couches ne soit fonction que du temps. Les modèles étudiés seront tous formés de deux couches superposées de fluides homogènes, incompressibles, non visqueux.

Il est possible, par des méthodes d'approximation successives, d'étudier dans des cas assez généraux l'influence des termes non-linéaires [3] mais ceci conduit à des développements vite inextricables. Le cas, intéressant lorsque les écoulements considérés sont engendrés directement ou indirectement par des phénomènes météorologiques ou astronomiques, où l'hypothèse de pression hydrostatique est acceptable, sera seul considéré ici. Alors, le système d'équations aux dérivées partielles non-linéaires homogènes d'ordre un, qui décrit les écoulements est hyperbolique, et les solutions par ondes simples peuvent être étudiées dans le plan de l'hodographe après intégration numérique des équations caractéristiques. Si l'on tient compte des forces de Coriolis, une telle simplification n'est plus possible et il faut se résoudre à intégrer numériquement les équations par la méthode des caractéristiques pour trouver les solutions à partir de conditions initiales données.

Il ne serait pas opportun, dans ce bref exposé, de détailler des calculs parfois laborieux. Nous nous sommes limités à dégager les résultats physiquement intéressants, en laissant au lecteur intéressé le choix de se reporter aux ouvrages cités en référence dans lesquels la mise en équations et les calculs sont explicités.

\section{I - Cas des écoulements hydrostatiques sans rotation}

En retenant toutes les hypothèses énumérées dans l'introduction, le système de deux équations aux dérivées partielles non-linéaires d'ordre un qui décrit les écoulements possibles, se réduit au système caractéristique correspondant de deux équations différentielles:

$$
\begin{aligned}
& \left(\frac{d U}{d H}\right)_{+}=-A_{+}(R, H, U) \\
& \left(\frac{d U}{d H}\right)_{-}=-A_{-}(R, H, U)
\end{aligned}
$$

(*) Contribution $n^{\circ} 252$ du Département Scientifique du Centre Océanologique de Bretagne. 

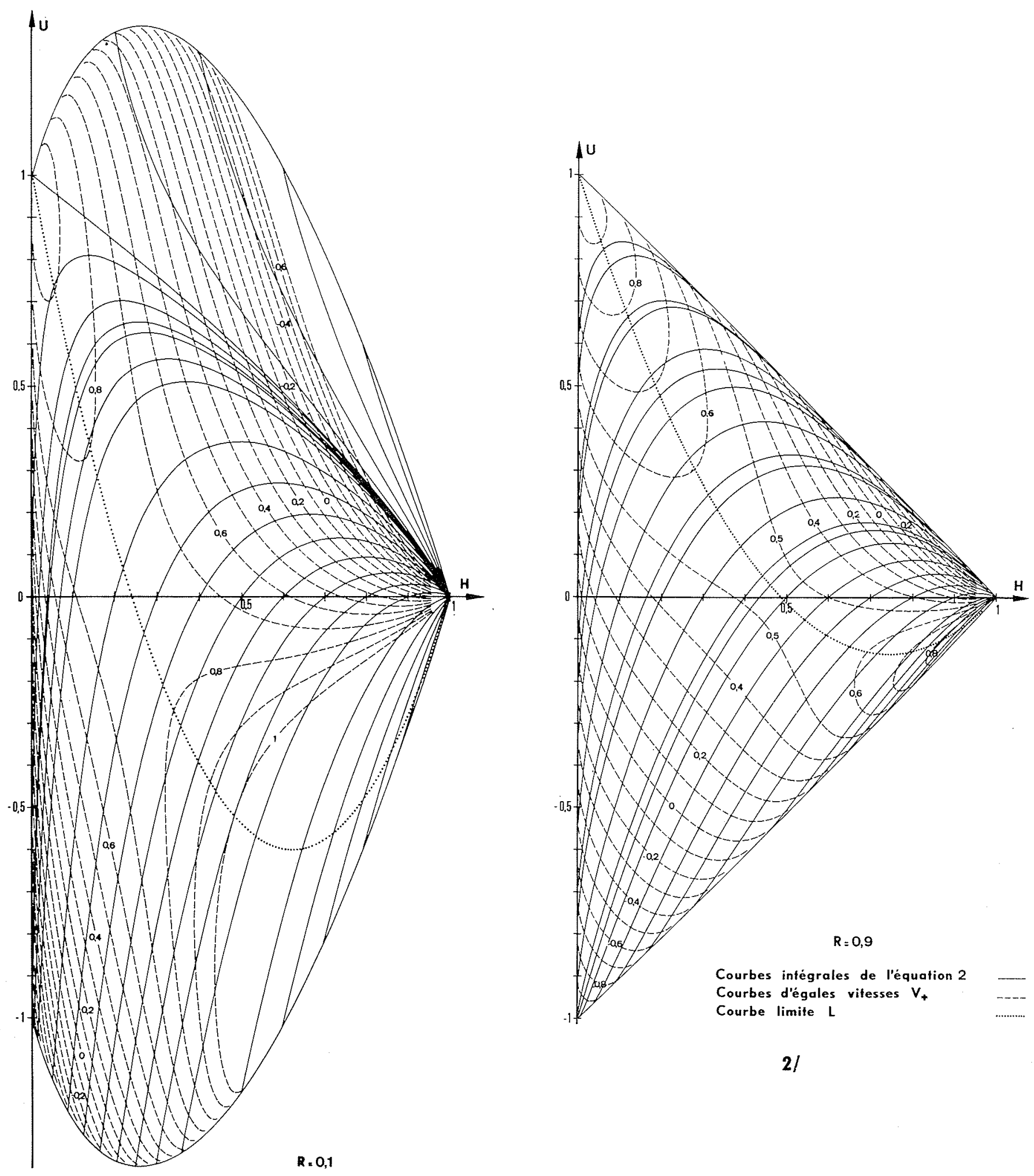

2/

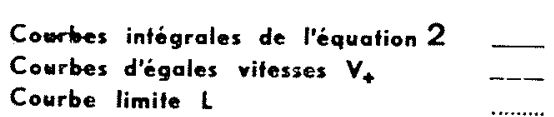


satisfaites respectivement sur les deux familles de courbes caractéristiques

$$
\begin{aligned}
& \left(\begin{array}{l}
d X \\
d T
\end{array}\right)_{+}=V_{+}(R, H, U) \\
& \left(\begin{array}{l}
d X \\
d T
\end{array}\right)_{-}=V_{-}(R, H, U)
\end{aligned}
$$

où

$$
\begin{gathered}
R=\rho_{1} / \rho_{2} \quad \mathrm{X}=x / D \quad T=t[(1-R) g / D]^{1 / 2} \\
\left.H=h / D \quad U=u_{1}[1-R) g D\right]^{-1 / 2}
\end{gathered}
$$

Les variables dimensionnées $g, x, t, \rho_{1}, \rho_{2}, h, u_{1}$ et $D$ sont respectivement l'accélération de gravité, les variables d'espace et de temps, les masses spécifiques dans la couche supérieure et inférieure, la profondeur de l'interface, la composante horizontale de la vitesse suivant $x$ dans la couche supérieure et la profondeur totale entre fond et plafond. Les expressions $A_{+}, A_{-}, V_{+}, V_{-}$sont des fonctions non-linéaires relativement compliquées [5].

Naturellement, le système d'équations (1) à (4) se prête à l'intégration numérique par la méthode des caractéristiques. Mais il est instructif d'étudier les solutions par «ondes simples » dans le plan de l'hodographe $(H, U)$, ce qui est possible puisque les équations différentielles (1) et (2) ne dépendent pas des variables $X$ et $T$. Une onde simple [6] est définie comme une perturbation se propageant sur l'une des familles de courbes caractéristiques dans une région où $H$ et $U$ étaient constants à l'instant initial de valeurs $H_{0}$, $U_{0}$, avant l'arrivée de la perturbation. Pour fixer le problème, considérons que la perturbation se propage sur la famille de caractéristiques $C_{+}$; dans ce cas, l'écoulement se fait dans le plan $(H, U)$ sur un segment de la courbe passant par le point $\left(H_{0}, U_{0}\right)$ et dont la pente est donnée par l'équation (2). L'équation (1) impose que $H$ et $U$ conservent des valeurs constantes sur chaque courbe caractéristique $C_{+}$et donc les courbes $C_{+}$sont des segments de droite, d'après l'équation (3).

Les figures 1 et 2 représentent, en trait continu, la famille des courbes obtenues par intégration numérique de l'équation (2) pour les valeurs 0,1 et 0,9 du paramètre $R$. Le domaine d'intérêt physique est borné par les conditions:

$$
\begin{aligned}
& H \geqslant 0 \\
& U<(1-H)\left(H R^{-1}+1-H\right)^{1 / 2} \\
& U>-(1-H)\left(H R^{-1}+1-H\right)^{1 / 2}
\end{aligned}
$$

Les deux dernières contraintes sont imposées par des conditions de stabilité de l'interface par rapport à de petites perturbations (Instabilité de Kelvin-Helmholtz).

Sur ces figures, sont aussi représentées, en traits discontinus longs, les courbes d'égales vitesses de propagation $V_{+}$ données par l'équation (3) et, en pointillés, les courbes limites $L$ qui ont pour équation :

$$
U=(1-H)^{2}-R^{-1 / 2} H(1-H)
$$

et séparent le domaine d'intérêt physique en deux régions.
En-dessous de cette courbe $L$, la vitesse $V_{+}$augmente avec $H$ en suivant les courbes intégrales en trait plein; au-dessus de la courbe $L, V+$ diminue lorsque $H$ augmente. Les ondes simples dans la zone au-dessus de $L$ ont un comportement analogue aux ondes de surface, tendant vers un ressaut lorsque la profondeur de l'interface est plus grande en amont qu'en aval de l'onde. Par contre, dans la zone en-dessous de $L$ qui correspond aux conditions océaniques, seuils des ressauts «inversés » seront possibles, la profondeur de l'interface étant plus grande en aval qu'en amont de l'onde.

Lorsque $R$ tend vers 1 , il est évidemment possible de faire l'hypothèse de Boussinesq. Les expressions $A \pm$ se simplifient considérablement et les intégrales des équations (1) et (2) peuvent être explicitées [2]. Dans ce cas, que nous ne détaillerons pas plus ici, il est possible de définir, connaissant seulement les valeurs $H_{0}$ et $U_{0}$ en amont de l'onde simple, la profondeur critique où la nature des écoulements changera, sans faire appel au calcul numérique.

\section{II - Le cas des \\ écoulements hydrostatiques en tenant compte des forces de Coriolis}

Dans ce cas, le système d'équations qui décrit les écoulements plans contient le paramètre sans dimension:

$$
F=f\left[(1-R) g D^{-1}\right]-1 / 2
$$

qui correspond au paramètre de Coriolis $f=2 \Omega \sin \phi$, où $\Omega$ est la rotation terrestre et $\phi$ la latitude. Les composantes normales au plan de l'écoulement des vitesses horizontales apparaissent dans les équations qui forment un système hyperbolique mais non homogène, ce qui interdit les solutions par ondes simples. Le système caractéristique correspondant comprend quatre équations différentielles nonlinéaires à satisfaire sur un réseau de quatre familles de courbes caractéristiques et il faut se contenter de solutions numériques à chaque problème posé [1].

\section{II.A - Cas des ondes internes engendrées par un déséquilibre initial de l'inferface}

On considère le cas particulier où l'interface, à l'instant initial, comprend deux portions rectilignes horizontales reliées entre elles par une demi-sinusoïde. Les vitesses sont identiquement nulles dans la couche supérieure et inférieure à l'instant initial. L'ensemble ainsi décrit va évoluer vers un nouvel état sous l'influence des forces de gravité et de Coriolis en créant un train d'onde de part et d'autre de la zone centrale.

Dans le modèle étudié, les valeurs numériques utilisées sont les suivantes :

$$
\begin{array}{lll}
h_{1}=100 \mathrm{~m} & h_{2}=50 \mathrm{~m} & L=20 \mathrm{~km} \\
D=4000 \mathrm{~m} & g=9,8 \mathrm{~m} / \mathrm{s}^{2} & R=0,999
\end{array}
$$




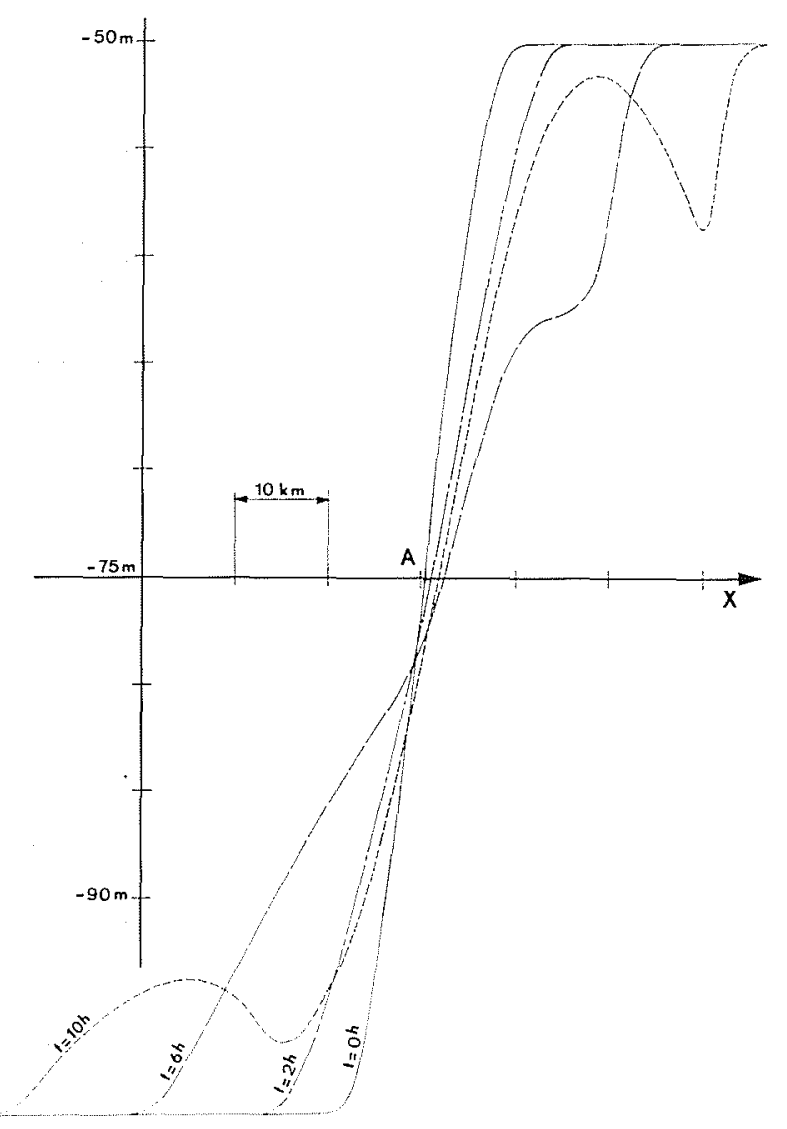

3/ Naissance des ondes aux abords de la perturbation initiale.

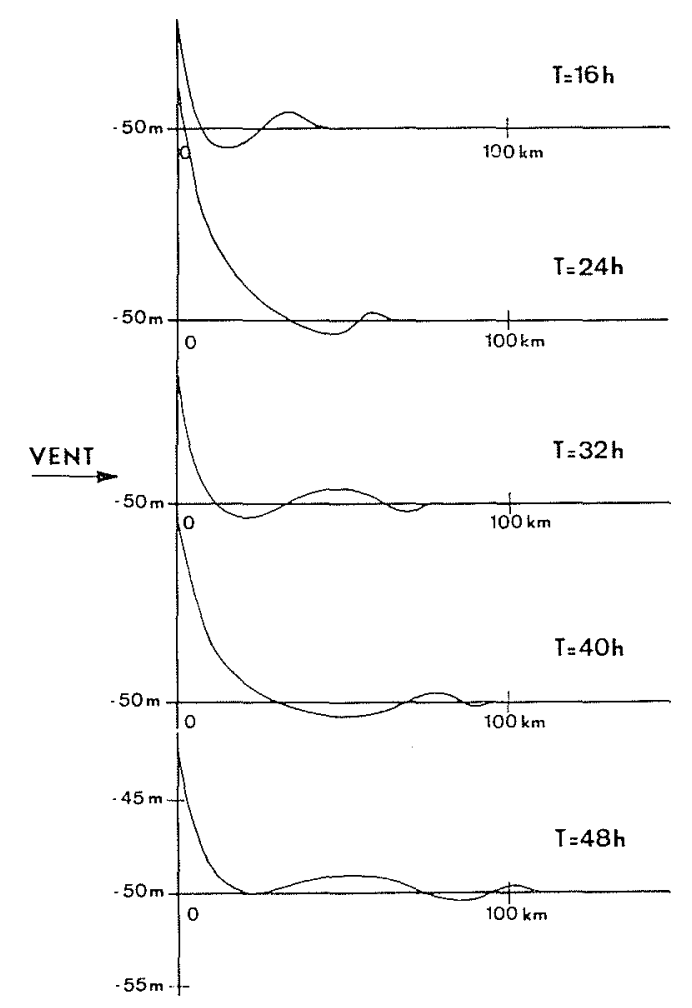

5/ Train d'ondes se propageant dans le sens du vent.

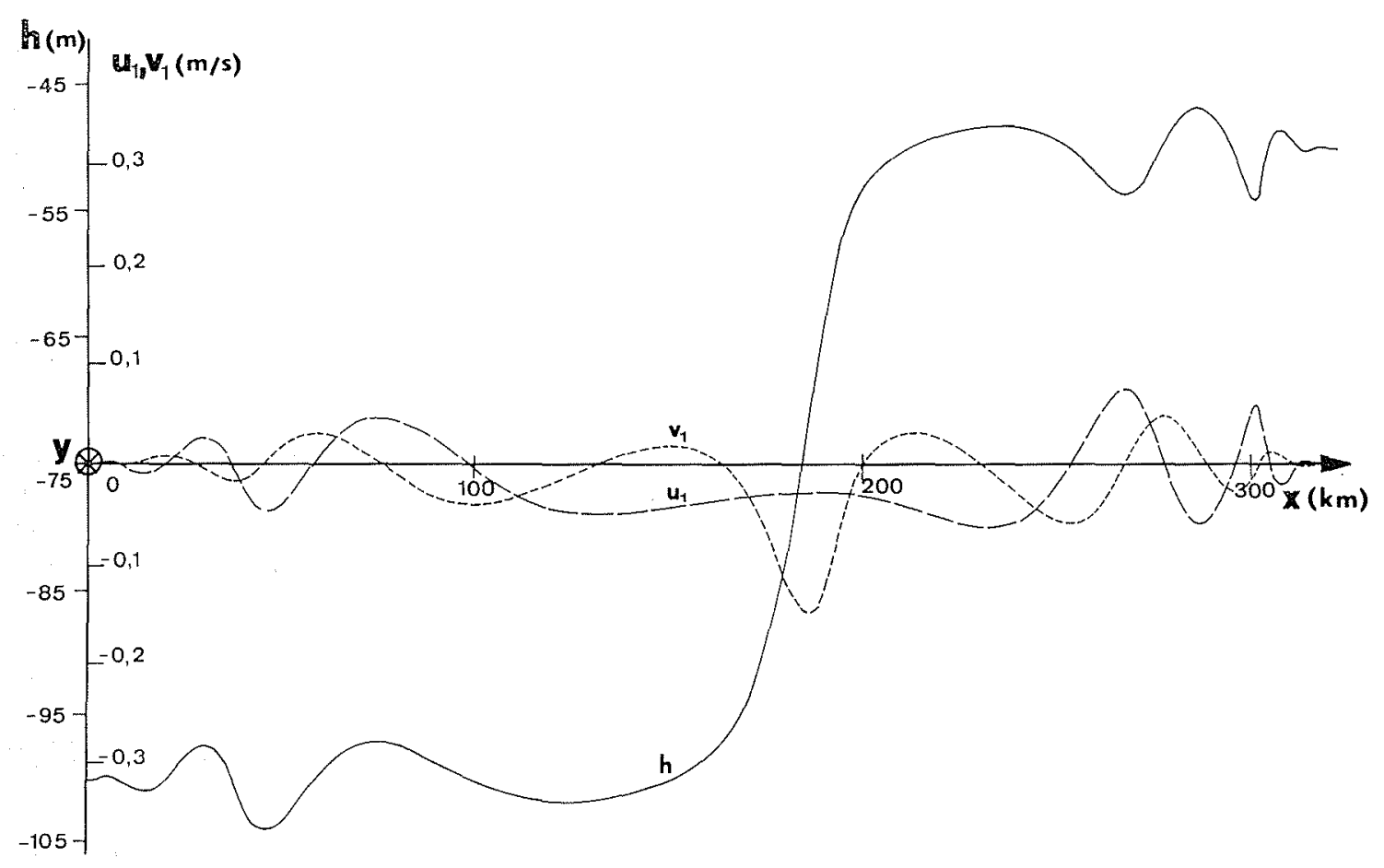

4/ Forme de l'interface et profil des vitesses à $t=48 \mathrm{~h}$. 


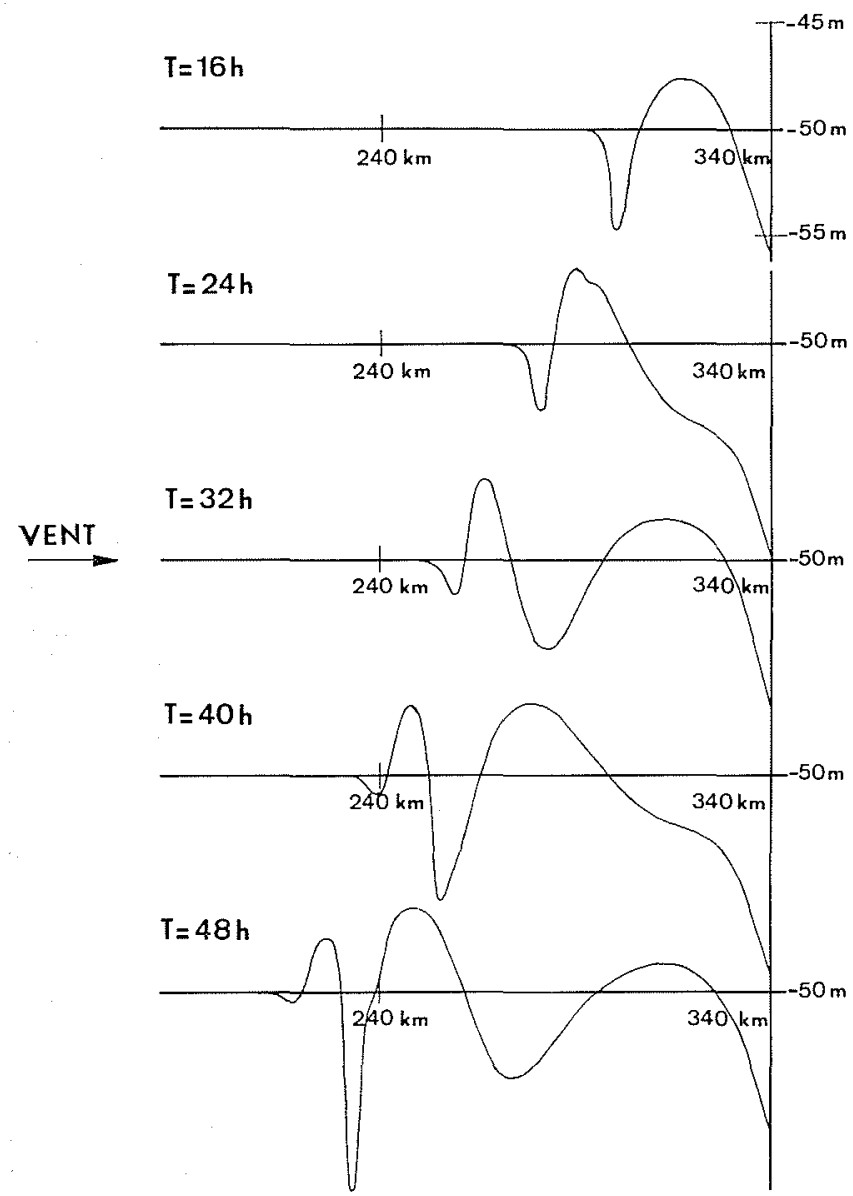

6/ Train d'ondes remontant le vent.

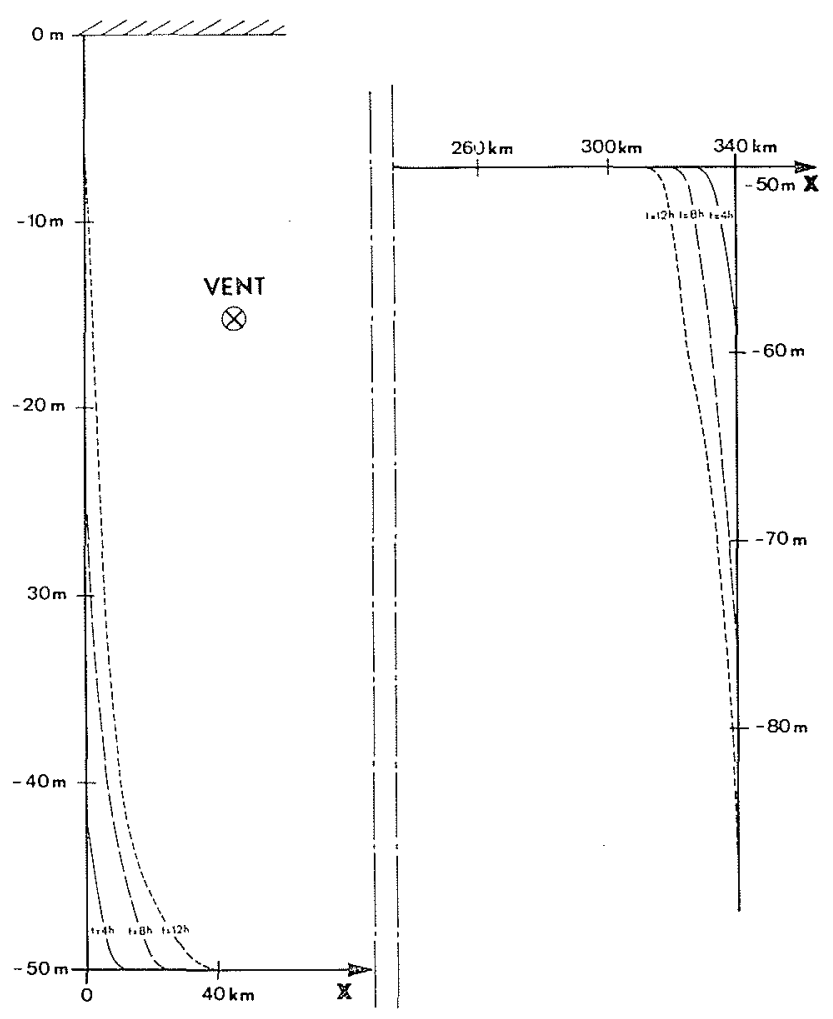

8/ Vent parallèle aux côtes.

Évolution de la forme de l'interface aux extrémités du bassin.

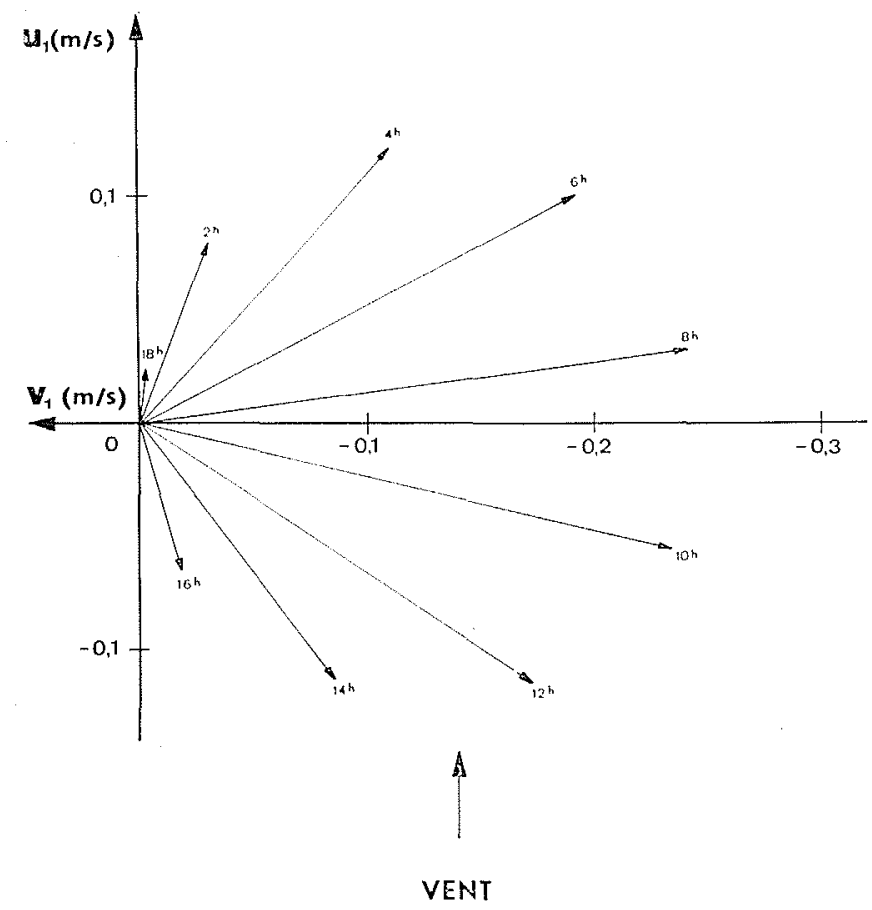

7/ Observation d'un courant d'inerlie dans la zone calme. 
La figure 3 permet de suivre la mise en mouvement des deux fronts d'ondes de part et d'autre de la perturbation initiale. Ils se propagent à des vitesses légèrement différentes de l'ordre de $3 \mathrm{~km} / \mathrm{h}$. Il apparaît nettement sur la courbe correspondante à $t=10 \mathrm{~h}$, que la pente du front se dirigeant vers la droite (front inversé) est plus raide que la pente du front progressant vers la gauche. Ceci illustre le mécanisme non-linéaire étudié au paragraphe précédent. La figure 4 montre l'écoulement à $t=48 \mathrm{~h}$; les trains d'ondes atteignent la limite du canal aux parois verticales et l'intégration numérique n'a pas été poussée plus loin. On constate que chaque oscillation des trains d'ondes possède une pseudo-longueur d'onde qui augmente de l'avant à l'arrière des trains. De même, l'étude des résultats en un point donné montre que les pseudo-périodes sont plus courtes que la période d'inertie et tendent vers celle-ci. Ces résultats sont explicables par le fait que, dans un système en rotation, la vitesse de groupe diminue et la longueur d'onde augmente pour une perturbation sinusoïdale lorsque la période tend en croissant vers la période d'inertie.

\section{II.B - Le cas d'ondes internes engendrées par le vent dans un bassin à deux couches}

Il est bien connu, sur la côte méditerranéenne, qu'un coup de vent peut provoquer un refroidissement soudain des eaux littorales. Ce phénomène s'explique par la remontée des eaux profondes plus froides que les eaux superficielles. Dans cette région, les observations océanographiques effectuées par le Laboratoire d'Océanographie Physique du Muséum National d'Histoire Naturelle, à bord de la bouéelaboratoire du CNEXO, ont permis d'observer la formation de courants d'inertie et d'ondes internes de périodes proches de la période d'inertie.

Un modèle théorique non-linéaire a été développé pour étudier ce type de phénomène. A l'instant initial, les deux couches fluides superposées sont au repos et l'interface horizontale. Sur ce bassin, long de $340 \mathrm{~km}$, souffle un vent de 30 nœuds. La tension superficielle $« c »$ due au vent, calculée par la formule d'Ekman, qui correspond dans ce cas à $0,6 \mathrm{~N} / \mathrm{m}^{2}$ est assimilée à une force par unité de masse appliquée à la couche supérieure et de valeur $c / p_{1} h$ sur la hauteur $h$ de celle-ci. Cette simplification respecte assez bien la réalité; on a en effet observé que les effets du vent se limitent à la couche superficielle, la thermocline jouant un rôle d'écran pour les échanges turbulents de quantité de mouvement [8].

La profondeur initiale de l'interface a été prise égale à $50 \mathrm{~m}$, la profondeur du bassin à $3000 \mathrm{~m}$ et $R=0,999$. Les figures 5 et 6 montrent l'évolution de l'interface près des extrémités du bassin lorsque le vent souffle perpendiculairement à la côte; la comparaison de ces courbes montre que les termes non-linéaires interviennent pour renforcer les ondes sur la côte vers laquelle souffle le vent et les atténuer sur celle d'où vient le vent. Dans la zone centrale du bassin, avant l'arrivée des ondes internes, on constate un courant d'inertie, figure 7 , qui correspond au courant du modèle d'Ekman.

Lorsque le vent est parallèle à la côte, on constate un phénomène d'upwelling et de downwelling très rapide, figure 8 ; dans ce cas, nous n'avons pas poussé l'intégration numérique du modèle au-delà de $t=12 \mathrm{~h}$, car des considérations physiques montrent qu'il s'applique mal à ces conditions. En effet, un courant littoral violent se produit alors dans une zone étroite, proche des côtes, et il faudrait tenir compte des forces de frottement latérales.

\section{Conclusion}

Le but de notre exposé était de montrer que les termes non-linéaires jouent un rôle non négligeable dans les écoulements à deux couches. Les quelques cas étudiés ne peuvent évidemment pas s'appliquer à tous les problèmes de l'ingénieur et de l'océanographe. Nous espérons seulement qu'ils encourageront ceux-ci à prendre en compte les termes non-linéaires, ou bien de s'assurer de leur influence négligeable dans chaque cas particulier rencontré.

\section{Bibliographie}

[1] ARHAN (M.). - Etude non-linéaire des ondes internes dans un milieu à deux couches fluides en rotation (1973). Rapp. Scient. Tech. CNEXO, n $\mathrm{n}^{\circ} 17$.

[2] Cavanié (A.). - Sur la genèse et la propagation d'ondes internes dans un milieu à deux couches (1969). Cahiers Océanographiques, XXI, nov. 1969, pp. 831-843.

[3] Cavanié (A.). - Modèle non-Linéaire et non-hydrostatique appliqué à l'étude des fronts internes dans le détroit de Gibraltar (1970). Cahiers Océanographiques, XXIII, juilletaoût 1971 , pp. 611-627.

[4] Cavanié (A.). - Observations de fronts internes dans le détroit de Gibraltar pendant la campagne océanographique OTAN 1970 et interprétation des résultats par un modèle mathématique (1972). Mém. Soc. Roy. Sc. Liège, 6 série, tome II, pp. 27-41.

[5] Cavanié (A.). - Etude non-linéaire des ondes internes dans un milieu à deux couches fluides sans rotation (1973). Rapp. Scient. Tech. CNEXO, $\mathrm{n}^{\circ} 16$.

[6] Courant (R.), Hilbert (D.). - Methods of mathematical physics, Vol. II. Interscience Pub., New York (1962).

[7] CRÉPon (M.). - Hydrodynamique marine en régime impulsionnel, $3^{e}$ partie : Océan à deux couches (1967). Cahiers Océanographiques, XXI, nov. 1969 , pp. 863-877.

[8] Gonella (J.), Crépon (M.), Madelain (F.). - Observations de courant, de vent et de température à la bouée-laboratoire (position A), sept.-oct. 1966 (1966). Cahiers Océanographiques, XXI, nov. 1969 , pp. 845-854.

[9] Phillips (O. M.). - «The dynamics of the upper ocean 》 (1969). Cambridge University Press.

[10] Ralston (A.), WILF (H. S.). - «Méthodes mathématiques pour calculateurs arithmétiques » (traduction de P. BÉRARD). Dunod, Paris (1965) 


\section{Discussion}

Président : M. le Professeur H. LACOMBE

M. le Président remercie M. ARHan de son exposé et suggère aux auditeurs de poser des questions sur le traitement de ce problème non linéaire par la méthode des caractéristiques.

En réponse à une question de $M$. Suberville, $M$. ArHan confirme que le terme $d \pi / d y$ est supposé nul et que, de ce fait, les ondes de surface sont à crêtes rectilignes horizontales.

M. Suberville souligne que les phénomènes exposés pas les auteurs, tels que : «ondes internes engendrées par un déséquilibre initial de l'interface » ou «ondes internes engendrées par le vent dans un bassin à deux couches », peuvent être étudiés expérimentalement sur la "plaque toumante» de l'Institut de Mécanique de Grenoble, où des recherches sont en cours sur le deuxième cas susvisé (thèse de M. RenounRD).

Un échange de vues s'instaure entre M. Renouard, M. Suberville et M. CRÉPon sur le terme de couplage - ou d'amortissement et de dissipation - entre les milieux 1 et 2 . Dans l'exposé qui a été présenté, il prend la forme du produit de $v$ par un terme de débit. Y-a-t-il un moyen d'appréhender v par des mesures in-situ?

M. Conntic intervient en ces termes :

Dans tous ces modèles, la seule possibilité d'une action d'un milieu sur l'autre est par le jeu de la pression. Dans cette optique on peut penser, en premier lieu, aux démonstrations du Professeur Woods, ce matin, qui mettaient en lumière - bien qu'il ne s'agisse pas des mêmes échelles - que dans la thermocline, sur des dizaines de mètres d'épaisseur, il existe des processus capables de transférer des quantités de mouvements horizontales; il y a donc nécessairement une action tangentielle de la couche supérieure sur la couche inférieure. D'autre part, ce qui m'a toujours frappé dans les enregistrements de M. Gonella, c'est le fait que, lorsqu'il se produit un coup de vent, on observe presque instantanément une très forte corrélation des effets jusqu'au niveau de la thermocline, alors que ce n'est qu'au bout d'un certain délai que se produit la mise en mouvement de la zone située en-dessous. Cela m'apparaît parfaitement logique, dans la mesure où le transfert de quantité de mouvement se fait par l'effet d'une «viscosité turbulente» qui doit disposer du temps nécessaire pour diffuser en profondeur l'énergie à travers la thermocline elle-même.
Je suis tout ì fait d'accord intuitivement sur le fait que, dans des problèmes impulsionnels de ce genre, les termes d'inertie considérés sont les termes prépondérants au premier ordre. Mais, à partir du moment où on recherche des approximations d'ordre supérieur, il me semble plus important de tenir compte des forces de frottement que d'introduire des termes d'inertie bealcoup plus compliqués.

M. Crépon, appuyé par M. GONELLA, rappelle les difticultés d'interprétations des mesures faites au courantomètre au niveau de la thermocline - parfois à une trentaine de mètres au-dessous de la surface - car on ignore souvent si cet appareil baigne dans le «milieu supérieur» ou le «milieu inférjeur». Se référant à l'intervention de $M$. CoAnTrC, il poursuit

Le schéma que j'ai proposé montre que, pour l'étude de la génération des courants, il faut tenir compte des propagations des ondes de gravité et que l'on ne peut bâtir - si l'on désire une bonne approximation - des théories ne mettant en jeu que le vent local, le courant local, etc; les transports dus aux propagations d'ondes de gravité, dépendent des variations spatiales du vent et, en particulier, du rotationnel du vent.

M. Chabert-D'Hières se déclare d'accord avec ce que vient de dire M. Crépon. Il signale que le processus décrit par $M$. Conntic a été observé dans l'expérience suivante faite à Grenoble par M. RENouARD dans un canal relativement long. On traîne un filet de $6 \mathrm{~m} \times 0,5 \mathrm{~m}$ sur la surface libre du fluide supéricur; on voit très bien la propagation vers le bas des phénomènes de turbulence ainsi artificiellement créés en surface; lorsque, au bout d'un certain temps, ces phénomènes arrivent au niveau de l'interface, celle-ci est perturbée et bientôt entrânéc.

M. le Président souligne l'intérêt de visualiser et de filmer le phénomène de transition que vient de décrire $M$. ChaberT-D'Hières.

Personne ne demandant plus la parole, M. le Président clôt la discussion, et en même temps la dernière Séance de la Session, en remerciant tous ceux qui en ont fait un dialogue intéressant et fructueux.

La séance est levée à $17 \mathrm{~h} 30$. 